\title{
Meteorological Station Remote Access (Emar)
}

\author{
Harold A. Rodríguez Arias, ${ }^{1}$, Moises Bolaño Davila. ${ }^{2}$, Genadiz Cardona Batista ${ }^{3}$ \\ Fundación Tecnológica Antonio de Arévalo, Centro de Investigaciones, Facultad de diseño e Ingeniería, Cartagena, \\ Colombia, harold.rodriguez@tecnar.edu.co \\ ${ }^{2}, 3$ Fundación Tecnológica Antonio de Arévalo, Facultad de diseño e Ingeniería \\ Cartagena, Colombia E-mail:moises.bolano@tecnar.edu.co, genadizcb@gmail.com
}

\begin{abstract}
Abstrac. Thanks to the intervention of technology, the rise of ICT, the massification of internet, with the use of sensors, electronics, and software has been able to establish the exact data climatic and atmospheric condition of a city; however, in a city climate state it is not the same due to different factors, growth and locations of various neighborhoods and districts of the city. It is important in the diagnosis climate strategy sectorizar the city and have more accurate data on different weather condition you can get in each location points is applied. The use of ICT tools in this project is vital because you want to let the hand of every citizen digital status information on climate, atmospheric pressure, wind speed and to the degree of contamination of a place ' $X$ ' in a city across the web, making it compatible with all electronic devices currently used as smartphones.
\end{abstract}

Keyword: Pollution, Climate, sectorize, Sensors, red, TIC.

Digital Object Identifier

(DOI):http://dx.doi.org/10.18687/LACCEI2016.1.1.222

ISBN: 978-0-9822896-9-3

ISSN: $2414-6390$

$14^{\text {th }}$ LACCEI International Multi-Conference for Engineering, Education, and Technology: "Engineering Innovations for Global Sustainability", 20-22 July 2016, San José, Costa Rica. 


\title{
Meteorological Station Remote Access (Emar)
}

\author{
Harold A. Rodríguez Arias, ${ }^{1}$, Moises Bolaño Davila. ${ }^{2}$, Genadiz Cardona Batista ${ }^{3}$ \\ ${ }^{1}$ Fundación Tecnológica Antonio de Arévalo, Centro de Investigaciones, Facultad de diseño e Ingeniería, Cartagena, Colombia, \\ harold.rodriguez@tecnar.edu.co \\ ${ }^{2,3}$ Fundación Tecnológica Antonio de Arévalo, Facultad de diseño e Ingeniería \\ Cartagena, Colombia E-mail:moises.bolano@tecnar.edu.co,genadizcb@gmail.com
}

\begin{abstract}
Abstrac. Thanks to the intervention of technology, the rise of ICT, the massification of internet, with the use of sensors, electronics, and software has been able to establish the exact data climatic and atmospheric condition of a city; however, in a city climate state it is not the same due to different factors, growth and locations of various neighborhoods and districts of the city. It is important in the diagnosis climate strategy sectorizar the city and have more accurate data on different weather condition you can get in each location points is applied. The use of ICT tools in this project is vital because you want to let the hand of every citizen digital status information on climate, atmospheric pressure, wind speed and to the degree of contamination of a place ' $X$ ' in a city across the web, making it compatible with all electronic devices currently used as smartphones.
\end{abstract}

Keyword: Pollution, Climate, sectorize, Sensors, red, TIC.

\section{INTRODUCCIÓN}

El impacto de las TIC en nuestros días, hace que cada proyecto que se va a realizar, se piense de una manera diferente, o mejor aún, siempre se piensa en ¿cómo será afectado por las TIC?, o que ¿cómo afectará el proyecto a las TIC?, este último es el caso en el que se debe trabajar, para aprovechar las TIC y masificar el alcance de cada proyecto.

El clima no escapa de ella, y aunque directamente no se ve cómo es que las TIC lo afecta, cada día vemos más aplicaciones que informan e incluso predicen el estado del clima, o se puede observar el clima de otra ciudad o país. El estado del clima es cambiante debido a muchos factores y fenómenos que lo afectan provocando esos grandes cambios impredecibles en muchos casos; Sin embargo es importante que como habitantes y directamente afectados por los cambios climáticos se tenga la posibilidad de saber cuál es la condición climática actual en cualquier lugar de la ciudad, en este caso de Cartagena, con esta aplicación se podría saber si se puede asistir a un lugar sin problemas debido a su condición atmosféricas.

En Cartagena, como en la mayoría de las ciudades del país se tiene un dato general del estado climático, pero no se tiene un dato exacto de un lugar específico en esa ciudad, es decir de un barrio, corregimiento, etc. Por ejemplo en Cartagena se tiene el estado climático de la zona ubicada alrededor del aeropuerto, que es donde está

Digital Object Identifier (DOI): http://dx.doi.org/10.18687/LACCEI2016.1.1.222 ISBN: 978-0-9822896-9-3

ISSN: $2414-6390$ la estación meteorológica que alimenta aplicaciones como AccuWeather ${ }^{1}$, una de las aplicaciones más usadas por los usuarios de Smartphone, pero en toda la ciudad el clima no es el mismo, debido a que el aeropuerto está más cerca de la vía al mar y los demás barrios de la ciudad no. Puede pasar y es muy frecuente que en algunas partes de la ciudad llueve y en otros lugares no, esto indica que la información no es exacta para las personas que observan desde las diferentes páginas o medios de comunicación el clima de la ciudad.

La finalidad de este proyecto es conceder esa oportunidad a la comunidad de poder consultar el estado climático y atmosférico en los alrededores de la Fundación Tecnológica Antonio de Arévalo a través de su sitio web; sin embargo cabe mencionar que posterior a esto también se sectorizará la ciudad de tal forma que se tengan los datos climáticos de los diferentes rincones de Cartagena, a través de la construcción de una malla de nodo sensores que abarquen cada uno de los barrios de la ciudad. En este proyecto solo se incluirán nodos sensores en las dos sedes de la Fundación Tecnológica Antonio de Arévalo ubicadas en los barrios centro y pie del cerro para realizar la fase de funcionalidad del sistema, sin descartar la ampliación del espectro de cobertura de toda la ciudad de Cartagena. Esta es el valor agregado que tiene el proyecto con respecto a otros, pues su base de datos se nutrirá de una red de nodo sensores que, como se dijo, registrará el estado del tiempo en los diferentes lugares de la ciudad y no a nivel global. Cabe anotar que este proyecto también se nutre de los servicios ofrecidos por la Red Nacional Académica de Tecnología Avanzada RENATA en materia de almacenamiento, procesamiento de datos y comunicación síncrona.

\section{DISEÑO Y DESARROLLO DE UNA ESTACIÓN METEOROLÓGICA DE ACCESO REMOTO (EMAR).}

Este prototipo de sistemas clasificado como nodo sensor es una solución para realizar una actividad específica como esta; sin embargo, el diseño de este prototipo y su efectividad en la recepción y transmisión de datos dependerán de la calidad del hardware y software utilizado. ${ }^{1}$ Aplicación para Smartphone o tabletas que muestra y predice el estado
del tiempo.

$14^{\text {th }}$ LACCEI International Multi-Conference for Engineering, Education, and Technology: "Engineering Innovations for Global Sustainability", 20-22 July 2016, San José, Costa Rica. 
En un primer momento para establecer el diseño de EMAR se tuvieron en cuenta los siguientes aspectos:

- Mostrar la temperatura, presión atmosférica, determinar si llueve o no, velocidad y dirección del viento y posiblemente un sensor de $\mathrm{CO} 2$, en un lugar específico de la ciudad.

- Accesibilidad de los datos a través de la web

- Obtención de datos en tiempo real

Estos aspectos fueron de gran ayuda para iniciar el proyecto EMAR, el cual está basado en un diseño sencillo pero eficaz desde el punto de vista electrónico y gráfico, para mayor facilidad del manejo de los datos por parte del usuario, ya que estará representado con el mapa de la ciudad de Cartagena y la ubicación de los sensores que se encontraran en los diferentes barrios de la ciudad.

Para llegar a este punto y obtener los resultados se establecieron unas fases a nivel de diseño en hardware y software para detallar de manera concreta el funcionamiento del sistema.

Fase de análisis. Durante el periodo de esta fase se observaron las variables que se medirán desde los sensores, además de realizar interrogantes, tales como: ¿de qué forma se van a mostrar los datos?, ¿cuán efectivo y preciso debe ser el sistema?, ¿cuál será el margen de error que va a tener el sistema?, ¿cómo se transmitirán y recibirán los datos?, ¿qué tipo de lenguaje de programación se va a utilizar?, y tener en cuenta las ventajas y desventajas del sistema, compatibilidad del sistemas con los diferentes aparatos electrónicos existentes, accesibilidad a la aplicación y manejo por parte de los usuarios.

Gracias a estos interrogantes se pudo obtener datos muy importantes para el perfecto diseño y acoplamiento de los diferentes sensores, y tener un funcionamiento óptimo del sistema. Se determinaron los siguientes aspectos:

Hardware: las tarjetas que controlan el sistema son marca Arduino $^{2}$, por su eficiencia, posibilidades de conexión y lenguaje de programación robusto, flexibilidad en el diseño y economía; por su precisión en medición se utilizaran los sensores de presión atmosférica, temperatura y humedad DTH22 y BMP085 [5], ya que su efectividad en intervalo de medición es de $0,1 \%$ los cuales dan una gran ventaja para la exactitud de los datos. Teniendo en cuenta lo dicho anteriormente, cabe resaltar que además de proporcionar datos importantes sobre el estado del tiempo, se anexará a cada uno de los módulos un medidor de CO2 para verificar el impacto ambiental de la ciudad en cada uno de los sectores, ya que hay zonas industriales que podrían estar afectando zonas aledañas a ellas, debido

\footnotetext{
2 Arduino es una plataforma de electrónica abierta para la creación de
prototipos basada en software y hardware flexibles y fáciles de usar. Se
creó para artistas, diseñadores, aficionados y cualquiera interesado en

2 Arduino es una plataforma de electrónica abierta para la creación de
prototipos basada en software y hardware flexibles y fáciles de usar. Se
creó para artistas, diseñadores, aficionados y cualquiera interesado en

2 Arduino es una plataforma de electrónica abierta para la creación de
prototipos basada en software y hardware flexibles y fáciles de usar. Se
creó para artistas, diseñadores, aficionados y cualquiera interesado en crear entornos u objetos interactivos.
} $14^{\text {th }}$ LACCEI International Multi-Conference for Engineering, Education, and Technology: "Engineering Innovations for Global Sustainability", 20-22 July 2016, San José, Costa Rica. a la propagación y dirección del viento. El sensor seleccionado para esta labor es CO2 BTA de vernier [8].

En cuanto a la transmisión de los datos desde los sensores hasta la base de administración, se implementarán tarjetas inalámbricas $x_{b e e^{3}}$ las cuales tienen un nivel de transmisión óptimo y eficiente para el desarrollo de este proyecto.

Software: de acuerdo a esos requerimientos que se obtuvieron después de haber realizado el análisis de todas las posibles situaciones que se puedan presentar; el lenguaje de programación a utilizar es $\mathrm{C}$ para trabajar a bajo nivel y en cuanto a la implementación grafica se trabajara con HTML para que sea compatible con la página web. En cuanto al manejo de las variables y procesos a utilizar con cada una de las tarjetas trae consigo se implementará el lenguaje de programación que trae por defecto como sucede con Arduino.

Fase de diseño. En cuanto al diseño del proyecto EMAR tiene dos partes, una de software y otra de hardware; en cuanto al software se mostraran los datos de forma gráfica a través de un mapa de la ciudad de Cartagena con la ubicación de cada uno de los sensores como se ve en la figura 1 .

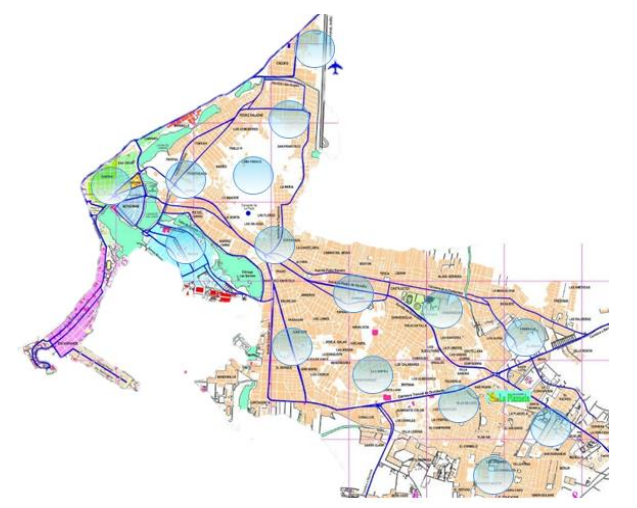

Figura 1. Mapa de Cartagena y posible ubicación de las EMAR

Cada uno de los círculos azules representa un sistema de dos nodos sensores con cobertura de $1 \mathrm{~km}$ en línea de vista cada uno y una estación base que es donde se recibirán los datos. Prácticamente este grupo de elementos conforman una estación base por sector, este sistema se podría representar como lo indica la figura 2.

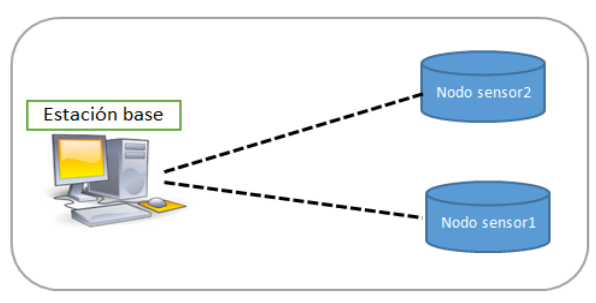

Figura 2. Estación base y nodos conectados.

3 Tarjeta inalámbrica especial para transmisión y recepción inalámbrica. 
Este sistema con interfaz gráfica le permitirá a cada uno de los usuarios que consulten los estados climáticos en ciertos puntos de la ciudad, identificando los sensores por las pequeñas bombillas de color rojo, al darle clic se verá la información necesaria del clima del lugar donde está ubicado el sensor, como se ve en la figura 5 .

Teniendo en cuenta lo anterior, EMAR debe tener un diseño de hardware que tenga engranaje con el software utilizado, porque es de gran importancia lograr la interacción de los dos sistemas. Es importante resaltar que cada nodo EMAR debe protegerse del clima en sí mismo, y es aquí donde se está trabajando en un sistema de protección de la electrónica, y se busca un material resistente a la intemperie, a las salinidad del ambiente y que a su vez no afecte ni las mediciones ni la transmisión de información, así como que garantice la no saturación de los sensores. En la figura 3 se aprecia un diseño de un nodo sensor por el momento resguardado en una caja plástica, usada en diseños eléctricos en exteriores.

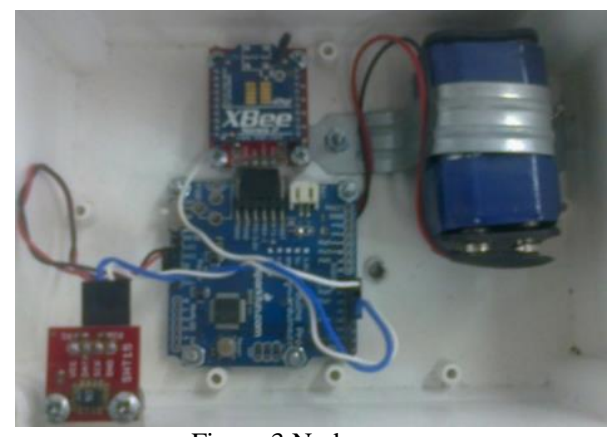

Figura 3 Nodo sensor

Fase de desarrollo. De acuerdo al progreso de este proyecto, se van desprendiendo las diferentes fases; en consecuencia con lo anterior, llega una parte de ejecución de EMAR que implica todo lo referente al acople del nodo sensor. Para poder realizar el ensamble de este proyecto, hay que tener en cuenta los pasos de configuración que traen las diferentes tarjetas y sensores; sin embargo, es importante el desarrollo de la aplicación para poder captar de manera eficaz y exacta la información o los datos obtenidos de los sensores. En cuanto a la aplicación como tal, se reflejará al usuario de manera agradable a través de una vista panorámica de lugares del país y del mundo como fondo y sobre estas imágenes se colocará toda la información requerida, como lo muestra la figura 4.

Para poder realizar la sectorización de la ciudad de Cartagena se necesita tener en cuenta la capacidad de cobertura que tiene cada uno de los sensores de acuerdo al sitio donde se coloque. Teniendo en cuenta lo anterior es importante resaltar que se estudiaron 2 opciones que podrían ser utilizadas para esta sectorización, como lo son el uso de repetidores RF y colocar un solo computador como receptor de todos los sensores y la otra es colocar sensores estratégicamente y su estación base cerca de los puntos de referencia.

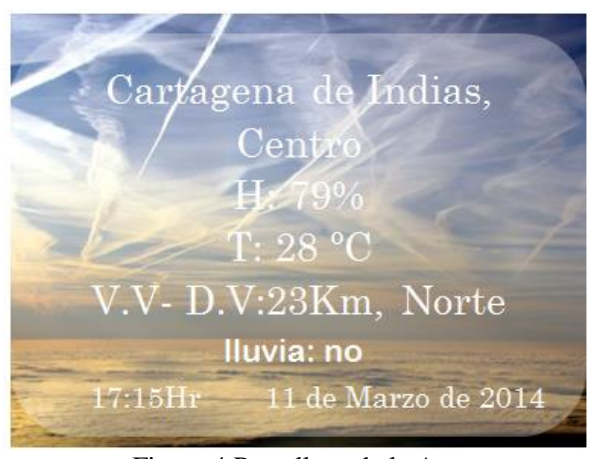

Figura 4 Pantallazo de la App

III SECTORIZACIÓN DE LA CIUDAD DE CARTAGENA PARA LA IMPLEMENTACIÓN DE LA ESTACIÓN METEOROLÓGICA DE ACCESO REMOTO (EMAR).

De acuerdo a los casos, se estipularon unos requerimientos para la sectorización que son los siguientes:

- La cobertura en $\mathrm{km}$ lineal que tiene cada sensor

- La interferencia de edificios demasiado altos

- Ubicación de las estaciones base (PC-Servidor)

- Transmisión y recepción de datos

- Posibles daños en los nodos sensores por la ubicación

Gracias a esto, se concluyó que se debían colocar los sensores en edificios altos de tal forma que las ubicaciones de las tarjetas inalámbricas les permitan realizar los envíos de los datos de forma eficiente. Además de esto se tuvieron en cuenta la ubicación de la ciudad con respecto a latitudes y altitudes para la adecuada ubicación de los sensores y no tener inconvenientes de visibilidad en $\mathrm{Km}$ a la redonda; sin embargo, este aspecto no conforma todo para la localización del sensor porque influyen los factores de infraestructura en los alrededores, que cortan el envío de la señal desde la tarjeta inalámbrica transmisora hasta la estación base donde se encuentra la tarjeta receptora, ver figura 5 .

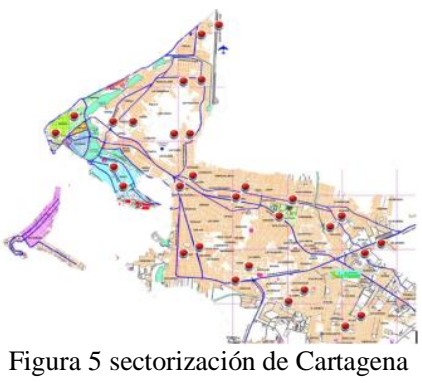

Figura 5 sectorización de Cartagena

$14^{\text {th }}$ LACCEI International Multi-Conference for Engineering, Education, and Technology: "Engineering Innovations for Global Sustainability", 20-22 July 2016, San José, Costa Rica. 


\section{IMPACTO DE (EMAR) EN LOS DIFERENTES SECTORES DE LA CIUDAD DE CARTAGENA.}

A través de un sistema como este, se le brinda la oportunidad a la comunidad de conocer sobre la realidad climática de la ciudad en todo momento y con un fácil acceso; la idea de colocar sensores de temperatura, humedad, presión atmosférica y viento, un sensor para medir $\mathrm{CO} 2$ es una gran ventaja, debido a que el viento es cambiante y su dirección puede variar en cualquier momento y arrastrar consigo alguna contaminación ambiental que pueda afectar a la comunidad y de esta manera se puede determinar qué sectores de la ciudad se ven afectados y tomar medidas de solución.

Por lo tanto, será una ventaja para las personas que dirigen este tipo de empresas poder tomar medidas correctivas respecto al nivel de concentración de $\mathrm{CO} 2$; cabe mencionar que con este proyecto se busca crear conciencia ciudadana en la comunidad, en cuanto a la contaminación, tala de árboles, que es lo que está causando actualmente algunos fenómenos ambientales y climáticos.

En cuanto al ámbito empresarial causara un impacto de tipo económico, debido a que podrán tener la oportunidad de saber el estado del clima en cualquier sector de la ciudad para prevenir inconvenientes logísticos dentro del desarrollo de sus actividades, como por ejemplo empresas de transporte de mercancía o de entrega de encomiendas, incluso los servicios domiciliarias.

En la última década este tipo de sistemas se han convertido en la nueva tendencia de medición debido a que permiten mayor capacidad de área de medición que los medidores normales, además de generar pocos gastos, sus sistemas son robustos y eficientes; sin embargo, han sido elaborados para poco consumo de ancho de banda.

Cabe mencionar que un punto a favor de este sistema es que se ha reemplazado el cableado por tarjetas inalámbricas, lo cual permite reducir los costos de inversión que se podrían tener con algunos otros instrumentos para mediciones. Por esta razón la infraestructura del sistema se vería en un proceso de simplificación de elementos y recursos, permitiendo la facilidad de uso en distintos campos de trabajo como: bodegas, áreas de cultivo, transportes, almacenes, etc.

\section{QUE MÁS OFRECE EMAR.}

Una de las características más importantes de EMAR es poder ofrecer información obtenida de los diferentes sensores a los usuarios, para esto se requiere almacenar la información de temperatura, humedad y CO para poder entregar las estadísticas y los reportes.

En el programa principal se puede observar (figura 6), los tres modos: estaciones, estadísticas y reportes.

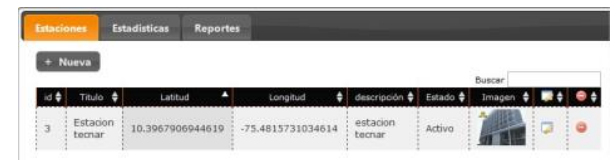

Figura 6. Pantallazo del programa modo administrador

En el primer modo, que es estaciones, se utiliza para administrar las diferentes estaciones, tanto para ubicarlas en el mapa, como para nombrarlas, ver figura 7.

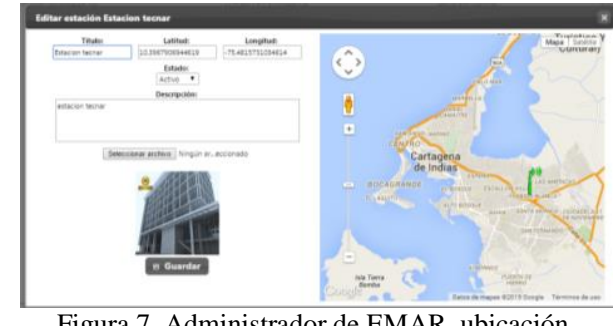

Figura 7. Administrador de EMAR, ubicación.

Una de las características principales es el modo estadísticas figura 8, que muestra en detalle por mes y días los datos de temperatura, humedad y $\mathrm{CO}$, ya es el usuario quien selecciona tanto la variable y la forma en que le muestre los datos, además en la parte inferior muestra el dato en tiempo real de la variable seleccionada, cabe resaltar que esta información se actualiza cada dos segundos.

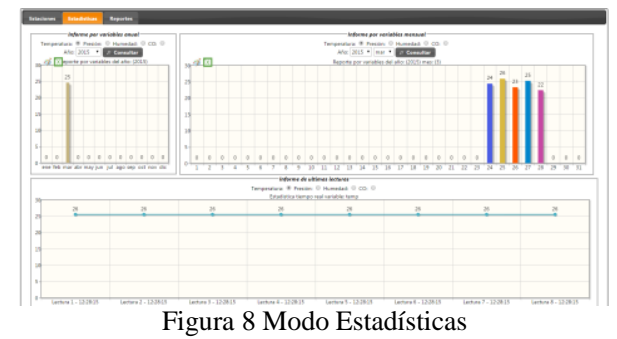

Al ubicar el cursor sobre una de las barras que muestran el promedio, ya sea del mes o de los días, saldrá un mensaje y este contiene la información del dato mayor y menor, incluso con la hora en la que se registró.

Por último se tiene el modo reporte, que entrega un informe detallado con información tomada cada dos segundos de cada una de las variables, figura 9, aquí el usuario podrá usar la información según su necesidad.

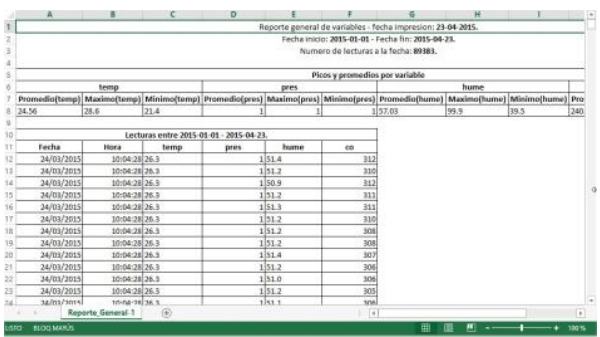

Figura 9. Modo reporte

$14^{\text {th }}$ LACCEI International Multi-Conference for Engineering, Education, and Technology: "Engineering Innovations for Global Sustainability", 20-22 July 2016, San José, Costa Rica. 
Lo que se pretende es almacenar la información de fecha hora y variable cada dos segundos con el fin de tener una muy buena base de datos y poder hacer predicciones a futuro con equipos con capacidad de supercomputo ofrecidos por la red académica nacional de tecnológica avanzada RENATA trabajando con su comunidad 3coA liderado en la ciudad de Cartagena de indias por el Ing. Jairo Serrano; permitiendo así tener una información exacta de las condiciones climáticas en cualquier lugar de la ciudad de Cartagena.

Por ejemplo para un mes se tiene que sacar el tiempo en segundos, cabe recordar que EMAR toma registros cada dos segundos, entonces, se debe multiplicar 30 días por 24 horas del día, por 60 minutos de la hora por 60 segundos del minuto, esto da 2’592.000 segundos, y como el sistema toma datos es cada dos segundos se debe dividir entre dos, para que el resultado sea 1'296.000 datos por cada variable, y como en este caso se está trabajando con tres esto da 3'888.000 datos, y cada uno de ellos está acompañado por la fecha y la hora.

A groso modo y haciendo la misma operaciones de arriba se tiene que para un año se requieren almacenar y poder entregar 47’304.000 datos para almacenar, con esta información se pretende generar un modelo matemático para la predicción del clima a futuro. Es por esto que es tan importante almacenar estos datos con el Servicio de computación en la nube para la actividad académica y científica que funciona sobre RENATA, por cada estación, lo que quiere decir ahora es que si tenemos 10 estaciones en toda la ciudad se multiplica por 10 el dato anterior y ese es el valor que se debe guardar, y la idea es que la estación trabaje por años y así tener una base de datos muy exacta del clima cada dos segundos, además anexándole otras variables como, presión atmosférica, velocidad de viento, dirección del viento entre otras, esto hará crecer la cantidad de información a almacenar y a trasmitir en el caso que se requiera un reporte de varios años.

En cuanto al aspecto económico cada nodo sensor tiene un costo aproximado de 700 dólares americanos, el costo de mantenimiento es bajo, sin embargo aún se deben hacer trabajos para evitar daños o malas lecturas debido al ambiente salino que tienen las ciudades costeras.

Proyectos de esta índole deben ser financiados por el gobierno nacional, ya que son de uso público y la base de datos nutrirá de información a entes de control o centros de investigación en el ámbito climático, ya que se podrá tener información detallada de múltiples variables en espacios de tiempos muy cortos, lo cual facilitará la construcción o simulaciones de mapas climáticos para nuestras ciudades, recordemos que al tener sensores distribuidos estos tomaran datos en tiempo real, sin importar la micro geografía cambiante debido al crecimiento y rápido cambio de las ciudades que alteran la dirección de los vientos dentro de las mismas.
Por último imagine estar conduciendo a un punto de destino y poder saber si al otro lado de la ciudad está lloviendo o en un sector, y poder reajustar su trayecto para evitar congestiones, $o$ en el peor de los casos inundaciones, sobre todo si se está circulando por una ciudad no muy conocida o por estar de paso en ella.

\section{AGRADECIMIENTOS}

Los autores quieren dar especial agradecimiento al Centro de Investigaciones Científicas y Tecnológicas CICTAR, de la Fundación Tecnológica Antonio de Arévalo TECNAR, por los aportes y asesorías en el proyecto.

También una mención especial a la Facultad de Diseño e Ingeniería, por la asesoría en lo que al conocimiento de redes y sensorica se refiere, cada uno de los profesores de la facultad aporto su granito de arena para este objetivo.

Y una atención muy especial al Ing. Marco Chico, coordinador académico de la RED RIESCAR para RENATA por su aporte en el proyecto para la utilización de los servicios de RENATA.

Y por último pero no menos importante agradecer al LACCEI 2016, porque abre una ventana para mostrar este trabajo al mundo.

\section{REFERENCIAS}

[1] Arduino. (2014). Recuperado de: http://www.arduino.ce/

[2] J. Del Río, Interoperabilidad en Redes de Sensores Para Monitorización Submarina, Editorial Académica Española, 2012-224 páginas.

[3] J. Garbarino, Protocolo para redes inalámbricas de sensores: Conciencia de energía y técnicas de diseminación en protocolos de red para redes inalámbricas de sensores, EAE, 2012-292 páginas.

[4] J. Sabaté. (4 de junio de 2013) Las cinco mejores aplicaciones gratuitas para saber el tiempo en Android. Recuperado

de: http://www.consumer.es/web/es/tecnologia/internet/20 13/06/04/216840.php

[5] J. Villarreal, F. Rodríguez, Sistema de Monitorización Ambiental Con Redes Inalámbricas de Sensores, Editorial Académica Española, 2012-120 páginas.

[6] National Instruments. (2014). Nodos de Medida de Redes Inalámbricas de Sensores (WSN). Recuperado de: http://sine.ni.com/nips/cds/view/p/lang/es/nid/206915 (25-02-2014)

[7] Universidad de Mendoza. Wireless Sensor Cloud for Agro-meteorological Studies. Recuperado de: https://sites.google.com/site/sensorcirrus/home (20-022014)

[8] Vernier. (2014). $\mathrm{CO}_{2}$ Gas Sensor. Recuperado de: http://www.vernier.com/products/sensors/co2-bta 